\title{
Estimation of channel parameters and background irradiance for free-space optical link
}

\author{
Afsana Khatoon, ${ }^{1, \star}$ William G. Cowley, ${ }^{1}$ Nick Letzepis, ${ }^{2}$ and Dirk Giggenbach ${ }^{3}$ \\ ${ }^{1}$ Institute for Telecommunications Research, University of South Australia, Mawson Lakes, SA 5095, Australia \\ ${ }^{2}$ Defence Science and Technology Organisation C3ID, Building 203, West Avenue, Edinburgh, SA 5111, Australia \\ ${ }^{3}$ Institute of Communications and Navigation, German Aerospace Center (DLR), Oberpfaffenhofen, Wessling 82234, Germany \\ ${ }^{*}$ Corresponding author: afsana.khatoon@mymail.unisa.edu.au
}

Received 28 November 2012; revised 27 February 2013; accepted 24 March 2013; posted 12 April 2013 (Doc. ID 180521); published 6 May 2013

\begin{abstract}
Free-space optical communication can experience severe fading due to optical scintillation in long-range links. Channel estimation is also corrupted by background and electrical noise. Accurate estimation of channel parameters and scintillation index (SI) depends on perfect removal of background irradiance. In this paper, we propose three different methods, the minimum-value (MV), mean-power (MP), and maximum-likelihood (ML) based methods, to remove the background irradiance from channel samples. The MV and MP methods do not require knowledge of the scintillation distribution. While the ML-based method assumes gamma-gamma scintillation, it can be easily modified to accommodate other distributions. Each estimator's performance is compared using simulation data as well as experimental measurements. The estimators' performance are evaluated from low- to high-SI areas using simulation data as well as experimental trials. The MV and MP methods have much lower complexity than the ML-based method. However, the ML-based method shows better SI and background-irradiance estimation performance. () 2013 Optical Society of America
\end{abstract}

OCIS codes: (200.2605) Free-space optical communication; (350.4600) Optical engineering.

http://dx.doi.org/10.1364/AO.52.003260

\section{Introduction}

Free-space optical (FSO) links are an attractive alternative to radio-frequency communication systems. They offer enormous unregulated bandwidth and license-free installation and are less prone to interference. However, their reliability is highly dependent on atmospheric and weather conditions. Absorption, scattering due to particulate matter in the atmosphere, and scintillation due to atmospheric turbulence can cause serious fading of the received signal $[1,2]$. In addition, usually the optical field is detected in the presence of background radiation, for instance from ambient light sources, the sun, or blackbody radiation. In outdoor FSO communications,

$1559-128 \mathrm{X} / 13 / 143260-09 \$ 15.00 / 0$

(C) 2013 Optical Society of America the background irradiation level changes slowly with time, altering the noise statistics at the receiver. An optical filter can reduce the background noise. However, background radiation that has same wavelength as the signal (or within the passband of the optical filters) will still accompany the detected optical signal. Detector noise (or shot noise), generated from the photodetection process causes internal interference. Circuit and electrical thermal noise are also produced or added in the process of photodetection and amplification. Background noise, shot noise and all electrical noise sources are often treated as an additive noise to the desired optical signal $[1,3]$.

Under weak atmospheric turbulence conditions the irradiance fluctuations obey log-normal statistics, and they obey exponential statistics in the very strong turbulence regime (saturation regime) [4]. In between these two extremes a plethora of 
irradiance distributions have been proposed including the Beckmann, $\mathrm{K}$, gamma-gamma [5-7] and M-distribution [8]. The Beckmann distribution results from the products of independent log-normal and Rician-distributed random variables [5]. However, its probability density function (PDF) can only be written in an integral form that has poor convergence properties, rendering it cumbersome for practical use. As a result, Al-Habash et al. proposed the gamma-gamma distribution to model the irradiance fluctuations from weak to strong atmospheric turbulence conditions [7]. The gamma-gamma distribution arises from the product of two independent gamma random variables and contains the K-distribution [9] as a special case (which is only valid in moderate to strong turbulence regimes).

Under certain atmospheric conditions, the parameters of the gamma-gamma distribution can be related to the Rytov variance, which in turn is dependent on atmospheric parameters such as the refractive-index structure parameter, inner/outer scale, and propagation path length [3]. In the work of Vetelino et al. the parameters of the gamma-gamma distribution were obtained by performing a best fit and maintaining the parameter relationship with the scintillation index (SI) [10]. Wang and Cheng propose a fractional moment-based shape parameter estimation of gammagamma distribution technique [11]. They also report that a maximum-likelihood (ML) parameter estimation technique for the gamma-gamma distribution is quite challenging as it requires the derivatives of the modified Bessel function with respect to (w.r.t.) both its argument and order-index [11].

In this paper we focus on the problem of estimating the background irradiation in the presence of a laser source corrupted by atmospheric scintillation. We propose two low-complexity methods that do not require knowledge of the underlying scintillation distribution: minimum-value (MV) and mean-power (MP) methods. The MV method, as the name suggests, simply takes the minimum sample value of a given observation interval. The MP method works by filtering out the scintillation fluctuations using a low-pass filter and adjusts the resulting signal via a background reference obtained from periodic off periods placed in the transmitted signal. In addition to the MV and MP methods, we propose an ML-based method that jointly estimates the background and the scintillation distribution parameters. To the best of our knowledge, all works on scintillation distribution parameter estimation do not consider the effects of background irradiance, or they assume its effects are removed by some other means. Usually, background has been measured experimentally at the beginning as well as the end of the experiment $[10,12,13]$. As the background irradiance can vary depending on weather and due to changes in cloud cover within 1-2 s, accurate estimation of background needs frequent measurements of background irradiance. Incorrect background removal can affect the channel parameter estimation as well as cause underestimation of the SI.
We compare each estimator's performance using simulated gamma-gamma scintillation plus background signals as well as real channel data collected from an experimental $20 \mathrm{~km}, 1550 \mathrm{~nm}$ optical link (as described in [14]). It was observed that the experimental data had significant fluctuations in background irradiance primarily caused by changes in cloud cover. To compare the estimator performance, we measure the mean bias error and root mean squared (RMS) error of the background estimate. In simulated channels, we demonstrate that the ML-based method has negligible bias and achieves an RMS error performance very close to the theoretical Cramer-Rao bound (CRB), which is a lower bound on the RMS error of any estimator. The other two methods showed significant bias and inferior RMS error performance. For real channel data, we find that the MV and ML-based methods have similar performance, whereas the MP method exhibits a significant error.

The remainder of this paper is organized as follows. In Section 2 we describe the FSO channel model for estimation. Section 3 presents the MV and MP methods. The ML-based method is described in Section 4. Section 5 compares the three methods using synthetically generated channel data, including constant-background and time-varying background cases. An estimator performance comparison using real channel data is presented in Section 6 . Concluding remarks are given in Section $\underline{7}$.

\section{Signal Model}

Consider an unmodulated laser beam propagating through a turbulent atmosphere. The optical energy of the laser beam as well as any background irradiation is collected by a receiver aperture at some distance from the laser source. We express the received irradiance signal collected by the receiver aperture at continuous time $t$ as

$$
I(t)=P x(t)+z(t)
$$

where $x(t)>0, \mathbb{E}[x(t)]=1$ denotes the fading due to scintillation, $z(t) \geq 0$ denotes the background irradiation, and $P>0$ denotes the average received optical power of the laser beam. We now assume the irradiance signal from Eq. (1) is converted to an electrical signal via photodetection and sampled at discrete time intervals of $T_{s}$ seconds. Note that $T_{s}$ is set to ensure the Nyquist rate is upheld w.r.t. the signals $x(t)$ and $z(t)$, and appropriate anti-aliasing low-pass filtering (LPF) is also assumed. Hence Eq. (1) at discrete time $n$ may be written as follows (for simplicity we have ignored constant scaling effects such as the photodetector efficiency):

$$
y[n]=P x[n]+z[n] .
$$

Note that in Eq. (2) we have assumed the received signal plus background power is much larger than 
any thermal or shot noise at the receiver, and hence thermal or shot noise effects are assumed to be negligible.

In practice, signals $x[n]$ and $z[n]$ are jointly temporally correlated random processes but with different coherence times. The fading due to scintillation typically has a coherence time on the order of milliseconds [3], whereas background irradiation has a coherence time on the order of seconds. Assuming the estimation observation interval $\left(T_{s} N \mathrm{~s}\right)$ is large enough to span many scintillation coherence time intervals but is also much smaller than the coherence time of the background irradiation, $z[n]$ can be considered constant and $x[n]$ fluctuates rapidly over the observation interval. Under these conditions we may rewrite Eq. (2) as

$$
y[n]=P x[n]+z,
$$

for $n=1, \ldots, N$. Assuming $T_{s}$ is greater than the coherence time of the scintillation, $x[n]$ and hence $y[n]$, $n=1, \ldots, N$, can be considered as a vector of independent and identically distributed (i.i.d.) random variables. Moreover, the PDF of $Y$ can be written as [15]

$$
f_{Y}(y)=\frac{1}{P} f_{X}\left(\frac{y-z}{P} ; \boldsymbol{\theta}\right) .
$$

for $y>z$, where $f_{X}(x ; \boldsymbol{\theta})$ denotes the scintillation PDF with parameters $\boldsymbol{\theta}=\left(\theta_{1}, \theta_{2}, \ldots\right)$.

The SI is an important measure related to the strength of the atmospheric turbulence (Rytov variance). For the random variable $X>0$ with mean $\mu_{X}$ and variance $\sigma_{X}^{2}$, the SI is defined as $[\underline{1}, \underline{16}]$

$$
\mathrm{SI}_{X} \triangleq \frac{\sigma_{X}^{2}}{\mu_{X}^{2}}=\frac{\mathbb{E}\left[X^{2}\right]}{\mathbb{E}[X]^{2}}-1
$$

Interestingly, the SI of the random variable $Y$ distributed according to Eq. (4) can be written in terms of $\mathrm{SI}_{X}$, namely

$$
\mathrm{SI}_{Y}=\mathrm{SI}_{X}\left(1+\frac{1}{\gamma}\right)^{-2}
$$

where $\gamma \triangleq(P / z)$ is defined as the signal-tobackground ratio (SBR). Thus we see that without taking the background irradiation into account, the measured SI will always underestimate the SI of the scintillation process, particularly for low SBRs.

\section{Distribution-free Background Estimation}

To begin with, we first describe two background irradiance estimation methods that do not require knowledge of the underlying scintillation distribution (hence the phrase distribution-free).

\section{A. MV Method}

Our first proposed method is called the MV method. Here we assume the estimation observation interval is set such that $z[n]$ can be considered constant, as in Eq. (3). An estimate of $z$ is obtained by selecting the MV over the observation interval, that is,

$$
\hat{z}=\min _{n=1, \ldots, N} y[n] .
$$

Thus the random variable $\hat{Z}=Y_{(1)}$ is the first-order statistic over the observation interval. We may therefore write the PDF of $\hat{Z}$ as [17]

$$
f_{\hat{Z}}(u)=N\left[1-F_{Y}(u)\right]^{N-1} f_{Y}(u) .
$$

While this estimator has low complexity and is independent of the scintillation distribution, it is straightforward to prove (via integration by parts)

$$
\mathbb{E}[\hat{z}-z]=P \int_{0}^{\infty}\left(1-F_{X}(x)\right)^{N} \mathrm{~d} x>0,
$$

where $F_{X}$ denotes the cumulative distribution function of $X$. We see that Eq. (9) is always greater than zero, and hence the estimator is biased. A biased estimator is generally considered undesirable in estimation theory [18]. Nonetheless, the bias reduces as $N$ increases, and as we shall see later Eq. (9) is useful as an initial estimate in our proposed ML-based estimation algorithm.

\section{B. MP Method}

Rather than setting an observation interval small enough to assume $z[n]$ is constant [as in Eq. (2)], the MP method first estimates the received laser power $P$ and then employs an LPF to remove the higher-frequency fluctuations due to scintillation. Note that this method also assumes the effect of low-frequency beam wandering can be neglected, as we were using large-divergence (near-spherical) beams ( $550 \mu \mathrm{rad}$ FWHM), which is far above any turbulent beam wander. The estimated power is then subtracted from the output of the LPF to obtain the background irradiation. To estimate the received laser power, the laser is switched off periodically for a short period of time (much smaller than the observation interval). Let $N=N_{\text {off }}+N_{\text {on }}$ be the observation interval with $N_{\text {off }} \lll N_{\text {on }}$. During the small off period, the background irradiation is assumed to be constant and is estimated via

$$
\hat{z}_{\text {off }}=\frac{1}{N_{\text {off }}} \sum_{n=1}^{N_{\text {off }}} y_{\text {off }}[n],
$$

where $y_{\text {off }}$ are the received samples corresponding to the off period. The received laser power is then estimated using

$$
\hat{P}=\frac{1}{N_{\text {on }}} \sum_{n=1}^{N_{\text {on }}} y_{\text {on }}[n]-\hat{z}_{\text {off }} .
$$

Finally the background irradiance during the on period is estimated using 


$$
\hat{z}_{\text {on }}[n]=g[n] * y_{\text {on }}[n]-\hat{P},
$$

where $g[n]$ denotes the coefficients of the LPF and * denotes the discrete convolution operation.

\section{ML-Based Method}

For the ML-based method, we assume the observation interval is small enough to assume the background can be considered constant and hence Eq. (3) is valid. From Eq. (4), the log-likelihood function can be written as [18]

$$
\begin{aligned}
\mathcal{L}(\boldsymbol{y} ; P, z, \boldsymbol{\theta}) & =\sum_{n=1}^{N} \log f_{Y}(y[n]) \\
& =-N \log P+\sum_{n=1}^{N} \log f_{X}\left(\frac{y[n]-z}{P} ; \boldsymbol{\theta}\right) .
\end{aligned}
$$

The ML estimates for $P, z$, and $\boldsymbol{\theta}$ are obtained by maximizing the log-likelihood function [Eq. (13)], that is,

$$
\left(\hat{P}_{\mathrm{ML}}, \hat{z}_{\mathrm{ML}}, \hat{\boldsymbol{\theta}}_{\mathrm{ML}}\right)=\arg \max _{P, z, \boldsymbol{\theta}} \mathcal{L}(y ; P, z, \boldsymbol{\theta})
$$

which can be done numerically using standard iterative optimization techniques $[19,20]$. Rather than performing the $L+2$ dimensional maximization problem in Eq. (14), we split the problem up into an $L$-dimensional and a one-dimensional subproblem, as described in Algorithm 1. Here, $L$ denotes the number of unknown parameters in the underlying distribution.

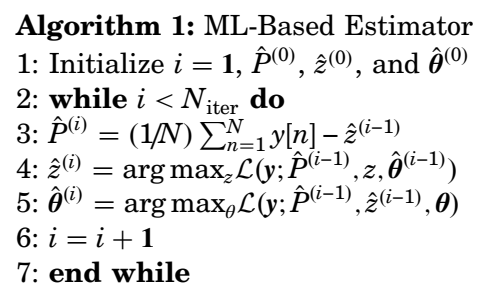

The two maximization problems in Algorithm 1 are dependent on $f_{X}(x ; \boldsymbol{\theta})$, and from this point on we assume $X$ is gamma-gamma distributed [7]:

$$
f_{X}(x ; \alpha, \beta)=\frac{2(\alpha \beta)^{\frac{(\alpha+\beta)}{2}}}{\Gamma(\alpha) \Gamma(\beta)} x^{\frac{\alpha+\beta}{2}} 1 K_{\alpha-\beta}(2 \sqrt{\alpha \beta x})
$$

where $\alpha$ and $\beta$ can be interpreted as the effective number of large-scale and small-scale cells of the scattering process, respectively [1]. We emphasize here that other distributions can be employed by following similar steps, described as follows.

\section{A. Estimation of Background Irradiation}

From Algorithm 1 we require the solution to

$$
\hat{z}=\arg \max _{z} \mathcal{L}(\boldsymbol{y} ; P, z, \boldsymbol{\theta}),
$$

assuming knowledge of $P$ and $\boldsymbol{\theta}$. This can be solved by finding where the slope of the log-likelihood function equals zero, namely

$$
\frac{\partial}{\partial z} \mathcal{L}(y ; P, z, \boldsymbol{\theta})=0 .
$$

Substituting Eq. (15) into Eq. (13) and taking the partial derivative w.r.t. $z$ yields

$$
\begin{aligned}
\frac{\partial}{\partial z} \mathcal{L}(y ; P, z, \alpha, \beta) & \\
= & -\left(\frac{\alpha+\beta}{2}-1\right) \sum_{n=1}^{N} \frac{1}{y[n]-z} \\
& -\sqrt{\frac{\alpha \beta}{P}} \sum_{n=1}^{N} \frac{1}{\sqrt{y[n]-z}} \frac{\mathrm{K}_{\alpha-\beta}^{\prime}\left(2 \sqrt{\frac{\alpha \beta}{P}(y[n]-z)}\right)}{\mathrm{K}_{\alpha-\beta}\left(2 \sqrt{\frac{\alpha \beta}{P}(y[n]-z)}\right)},
\end{aligned}
$$

where from the properties of Bessel functions [21, section 9.6.26],

$$
\mathrm{K}_{\nu}^{\prime}(a)=\frac{d}{d a} \mathrm{~K}_{\nu}(a)=-\frac{1}{2}\left(\mathrm{~K}_{\nu-1}(a)+\mathrm{K}_{\nu+1}(a)\right) .
$$

Therefore $\hat{z}^{(i)}$ in Algorithm 1 is the solution to

$$
\frac{\partial}{\partial z} \mathcal{L}\left(y ; \hat{P}^{(i-1)}, z, \hat{\alpha}^{(i-1)}, \hat{\beta}^{(i-1)}\right)=0,
$$

which can now be found numerically using Eq. (18), for instance via a bisection algorithm.

\section{B. Estimation of Scintillation Distribution Parameters}

From Algorithm 1 we require the solution to

$$
\hat{\boldsymbol{\theta}}=\arg \max _{\boldsymbol{\theta}} \mathcal{L}(\boldsymbol{y} ; P, z, \boldsymbol{\theta}),
$$

where $\boldsymbol{\theta}=(\alpha, \beta)$, assuming $P$ and $z$ are known. To solve the above problem we resort to numerical optimization techniques. In particular we employ the damped quasi-Newton-Raphson (NR) method $[19,20]$ with the Broyden-Fletcher-Goldfarb-Shanno ( $\overline{\mathrm{BF}} \overline{\mathrm{GS}})$ Hessian approximation as described in [19]. For convenience, we summarize this method in Algorithm 2 in Appendix A. The main advantage of this method is that it does not require the second partial derivatives of the log-likelihood function, which is problematic due to the modified Bessel function of the second kind in Eq. (15). All we require are the first derivatives w.r.t. $\alpha$ and $\beta$. Moreover, due to the symmetry between $\alpha$ and $\beta$ (i.e., they are interchangeable), 
we need only consider the derivative w.r.t. $\alpha$, which is given by

$$
\begin{aligned}
& \frac{\partial}{\partial \alpha} \mathcal{L}(y ; P, z, \alpha, \beta) \\
& =\frac{N}{2}\left(1+\frac{\beta}{\alpha}+\log (\alpha \beta)\right)-N \psi(\alpha)+\frac{1}{2} \sum_{n=1}^{N} \log \left(\frac{y[n]-z}{P}\right) \\
& \quad+\sum_{n=1}^{N} \frac{\frac{\partial}{\partial \alpha} \mathrm{K}_{\alpha-\beta}\left(2 \sqrt{\frac{\alpha \beta}{P}(y[n]-z)}\right)}{\mathrm{K}_{\alpha-\beta}\left(2 \sqrt{\frac{\alpha \beta}{P}(y[n]-z)}\right)},
\end{aligned}
$$

where $\psi(u)=\left(\Gamma^{\prime}(u) / \Gamma(u)\right)$ denotes the Digamma function [21, section 6.3.1]. From Eq. (22) we require the derivative of a Bessel function w.r.t. to its order. To evaluate this function we use the following result, which was obtained using [21, sections 9.6.24 and 9.6.26].

Theorem 1. Let $s(\nu)$ be a real, differentiable function of $\nu \in \mathbb{R}$ with first derivative $s^{\prime}(\nu)=(d / d \nu) s(\nu)$. Then

$$
\frac{d}{d \nu} \mathrm{K}_{\nu}(s(\nu) x)=s^{\prime}(\nu) x \mathrm{~K}_{\nu}^{\prime}(s(\nu) x)+\eta_{\nu}(s(\nu) x),
$$

for $|\angle s(\nu) x|<(1 / 2) \pi$ where $K_{\nu}^{\prime}(a)$ is defined as in Eq. (19) and

$$
\eta_{\nu}(a)=\int_{0}^{\infty} u e^{-a \cosh (u)} \sinh (\nu u) \mathrm{d} u .
$$

To the best of our knowledge a closed-form expression for Eq. (24) does not exist. However, its integrand is a well-behaved function, and the integral can be computed straightforwardly using standard numerical methods.

\section{Initial Parameter Estimates}

NR optimization is based on approximating the objective function by a tangent at the point of interest. As such, convergence is highly dependent on how close the initial estimates are to the global solution. To initialize the $z$ and $P$ estimate, we use

$$
\begin{gathered}
\hat{z}^{(0)}=(1-\rho) \min (y[1], \ldots, y[N]), \\
\hat{P}^{(0)}=\frac{1}{N} \sum_{n=1}^{N} y[n]-\hat{z}^{(0)},
\end{gathered}
$$

where $0<\rho<1$ ensures Eq. (18) does not become numerically unstable. In particular, we find setting $\rho=0.1$ gives good convergence behavior.

To initialize the scintillation distribution parameter estimates for $\alpha$ and $\beta$, we first estimate the SI:

$$
\hat{\mathrm{SI}}=N \frac{\sum_{n=1}^{N}\left(y[n]-\hat{z}^{(0)}\right)^{2}}{\left[\sum_{n=1}^{N}\left(y[n]-\hat{z}^{(0)}\right)\right]^{2}}-1 .
$$

Then we use the relationship between SI and the gamma-gamma distribution parameters, namely [7]

$$
\mathrm{SI}=\frac{1}{\alpha}+\frac{1}{\beta}+\frac{1}{\alpha \beta},
$$

and therefore in Eq. (22) use the substitution

$$
\beta=\frac{1+\alpha}{\alpha \hat{\mathrm{SI}}-1}
$$

to perform a one-dimensional root search to find $\alpha^{(0)}$, that is, the solution to

$$
\frac{\partial}{\partial \alpha} \mathcal{L}\left(y ; \hat{P}^{(0)}, \hat{z}^{(0)}, \alpha, \frac{1+\alpha}{\alpha \hat{\mathrm{S} I}-1}\right)=0,
$$

for $\alpha>\hat{\mathrm{S}} \mathrm{I}^{-1}$. The initial estimate $\beta^{(0)}$ can then be found using Eq. (29).

\section{Simulation Results}

\section{A. Constant Background Irradiation}

To verify our ML-based estimator, we compare its RMS error performance with the CRB, which is a lower bound on the variance of any estimator $[18,19]$. As a closed-form expression for the CRB is intractable, we resort to numerical methods for its computation; for instance, to evaluate the CRB for $\alpha$ we compute

$$
\operatorname{crb}(\alpha)=\left(\mathbb{E}\left[\left(\frac{\partial}{\partial \alpha} \mathcal{L}(y ; P, z, \alpha, \beta)\right)^{2}\right]\right)^{-1}
$$

where the expectation is over $y$ given $P, z, \alpha$, and $\beta$. (Note that in computing the CRB for a particular parameter we assume perfect knowledge of all other parameters.)

The RMS error of our estimator is computed via Monte Carlo simulations; that is, for each trial we randomly generate a vector of $N=1000$ i.i.d. gamma-gamma samples and then add an arbitrary constant background according to Eq. (3). We then estimate the parameters using Algorithm 1 and compute the estimation error. This procedure is repeated 1000 times to determine the RMS error. We also compare our ML-based method to the method-ofmoments estimators of $[11,22](\mathrm{MOM}-\mathrm{GG})$ and the maximum-likelihood estimator (ML-GG) of [22] that do not take the background irradiance into account.

Figure 1 compares the RMS error performance for $\alpha$ and $\bar{\beta}$ with their respective CRBs. We see that our ML-based algorithm achieves the CRB, whereas the other methods are not able to cope with the background irradiation. We also observed that the RMS error of the background irradiation also 


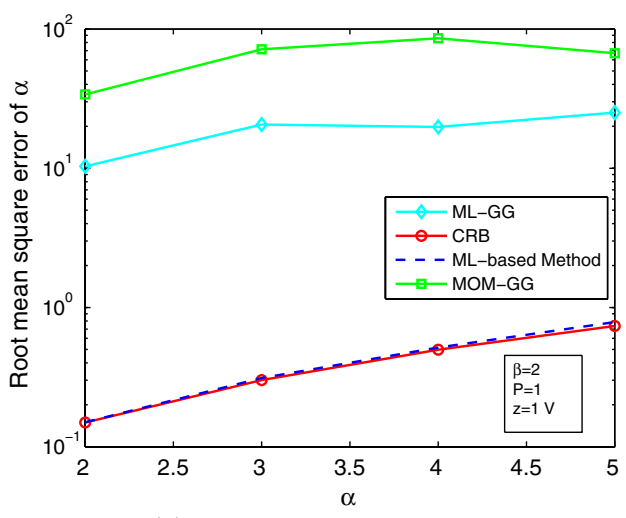

(a) RMS error for $\alpha$ with $\beta=2$.

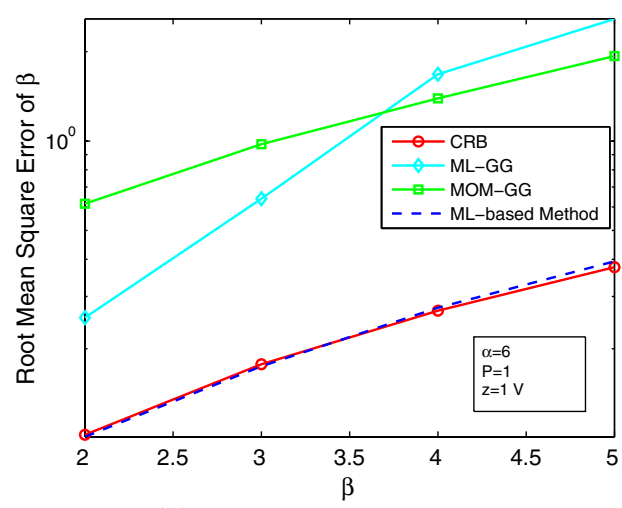

(b) RMS error for $\beta$ with $\alpha=6$

Fig. 1. Estimator RMS error performance comparison with $P=1, z=1$, where the solid curve with circle markers shows the CRB, dashed curve shows the ML-based method, the solid curve with diamond markers shows the ML-GG method [22], and the solid curve with square markers shows the MOM-GG method [11]. (a) RMS error for $\alpha$ with $\beta=2$ and (b) RMS error for $\beta$ with $\alpha=6$.

achieved the CRB, but we have omitted these results due to space limitations.

\section{B. Time-Varying Background Irradiation}

In practice, the background irradiation is a slow time-varying process. To evaluate each estimator's tracking performance, we model the time-varying background irradiation using a simple sinusoid, namely

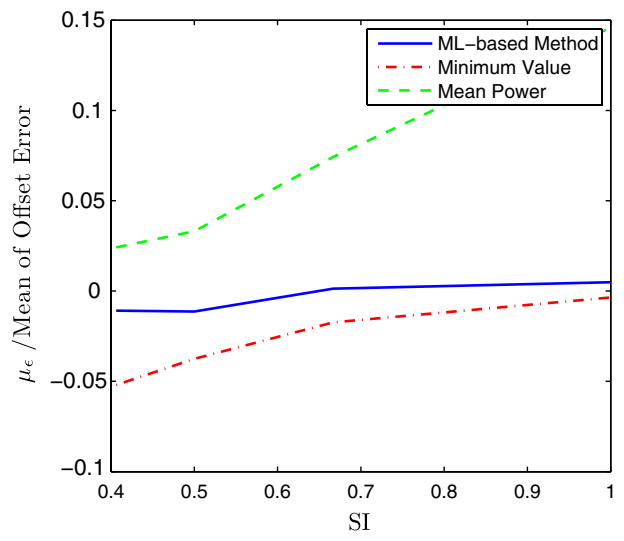

(a) Mean Bias Error

$$
z[n]=\tilde{z}+b \sin \left(2 \pi \frac{F}{F_{s}} n\right)
$$

where $\tilde{z}>0$ is the fixed background value, $b<\tilde{z}$ is the magnitude, $F$ is the sinusoid frequency, and $F_{s}$ is the sampling frequency. While this model does not reflect what occurs in practice, it provides a simple means to alter how rapidly the background

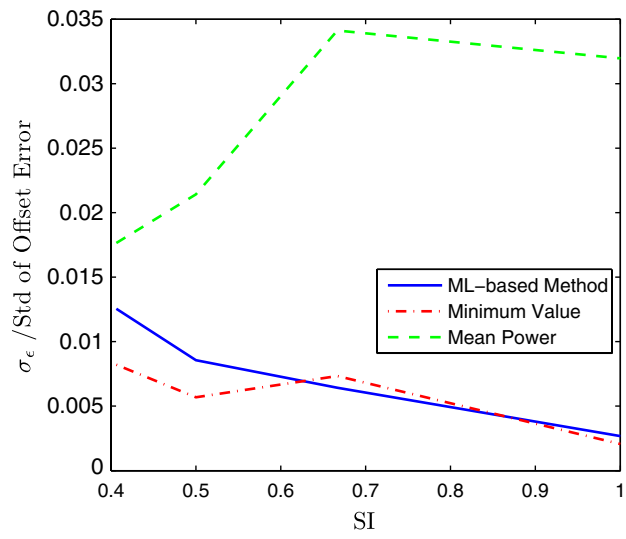

(b) RMS Error

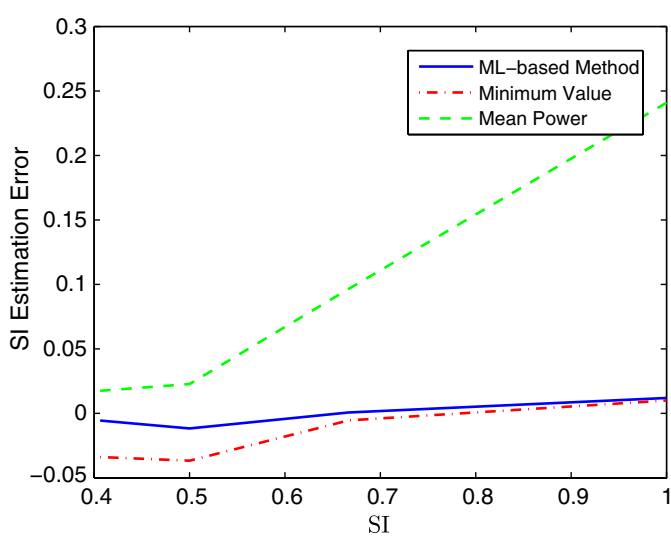

(c) Mean SI Estimation Error

Fig. 2. Comparison of estimation methods for simulated time-varying sinusoidal background irradiance: solid curve, ML-based method; dotted-dashed curve, MV method; and dashed curve, MP method. (a) mean bias error, (b) RMS error, and (c) mean SI estimation error. 
irradiation changes with time. Once $z[n]$ is constructed, $y[n]$ is simulated according to Eq. (2) with each $x[n]$ drawn randomly i.i.d. from a gammagamma distribution. Using these synthetically generated data, we then compare the estimators described in Sections 3 and 4 . Note that in order to evaluate the MP method, we also simulate the periodic intervals corresponding to when the laser is switched off.

We analyze the estimator performance for various levels of the SI. In particular, we calculate the bias or mean $\mu_{\epsilon}=\mathbb{E}[z[n]-\hat{z}[n]]$ and standard deviation $\sigma_{\epsilon}=$ $\sqrt{\operatorname{var}[z[n]-\hat{z}[n]]}$ of the background estimation error for the three estimators. In addition, we estimate the SI after removing the background and compute the SI estimation error.

A Monte Carlo simulation was performed using $F_{s}=1.0 \mathrm{kHz}, \tilde{z}=0.5, b=0.02$, and $F=0.25 \mathrm{~Hz}$ for SI over the range 0.4-1.0. For each trial of the Monte Carlo simulation, we generate $20 \mathrm{~s}$ (or 20,000 samples) worth of data. We set the observation interval of our estimators to be $2 \mathrm{~s}$ (or $N=2000 \mathrm{sam}$ ples). The $\mu_{\epsilon}$ and $\sigma_{\epsilon}$ of background estimation error for the proposed three estimators are shown in Fig. 2(a) and Fig. 2(b), respectively. Figure 2(a) shows that the ML-based method has lower $\mu_{\epsilon}$ error compared to the MV and MP methods. We see that the MV method and MP method over- and underestimate the background irradiation, respectively, whereas the ML-based method shows negligible bias.

The RMS error for the ML-based method is slightly higher than the MV method at low SI but shows similar performance at high SI, whereas the MP method has much higher $\sigma_{\epsilon}$ error compared to the other two methods. We find that the MV method performs better in terms of estimation bias in moderate to strong turbulence regimes. This is due to increased variability of the irradiance fluctuations about its mean, which increases probability of a very-lowirradiance fluctuation occuring, whereas under weak turbulence, the scintillation does not fluctuate much from its mean value, which is comparatively much higher than the background offset. Therefore there is a much lower probability that a low-irradiance fluctuation will occur.

The MV and MP methods have much lower computational complexity than the ML-based method. However, the MP method requires calculation of the MP from the obstructions or a background reference. In addition, the MV and MP methods are biased estimators, whereas the ML-based method is asymptotically unbiased and achieves the CRB.

We find the ML-based and MV methods need 1-2 s averaging of channel samples. The MP method needs a higher averaging period. Ideally this should be less than the rate of background variations, which is obviously difficult to achieve if the background is varying rapidly. For all methods, particularly the MP method, there is a tradeoff between tracking performance and the RMS background estimation error.
Setting $N$ small results in good tracking ability but poor RMS error performance and vice versa.

\section{Experimental Results}

We employed the three methods described in Sections $\underline{3}$ and $\underline{4}$ to remove the background irradiance from experimental irradiance data. These data was collected from a $20 \mathrm{~km}$ link between Mount Lofty

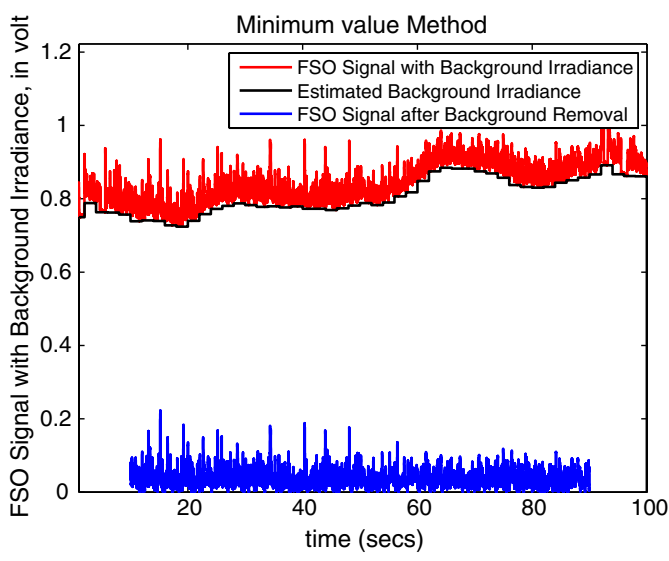

(a) MV Estimation of Background Irradiance.

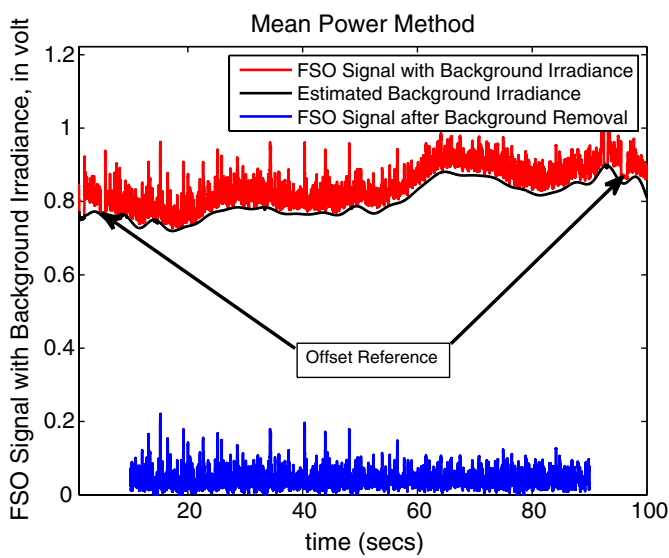

(b) MP Estimation of Background Irradiance.

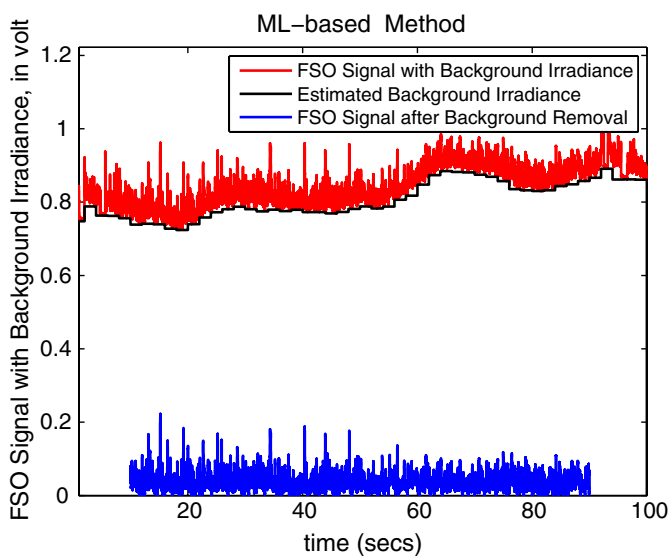

(c) ML Estimation of Background Irradiance.

Fig. 3. Estimation of background irradiance from experimental data. 
Table 1. Estimation Error Statistics with Experimental Data

\begin{tabular}{lcccccc} 
& \multicolumn{2}{c}{$\mu_{\epsilon}$} & & \multicolumn{2}{c}{$\sigma_{\epsilon}$} & \\
\cline { 2 - 3 } Method & Before & After & & Before & After & SI \\
\hline MP & 0.0045 & -0.0145 & & 0.0141 & 0.0185 & 0.6043 \\
MV & 0.0001 & -0.0138 & 0.0081 & 0.0179 & 0.9046 \\
ML-based & 0.0000 & -0.0138 & 0.0081 & 0.0176 & 0.7894 \\
\hline
\end{tabular}

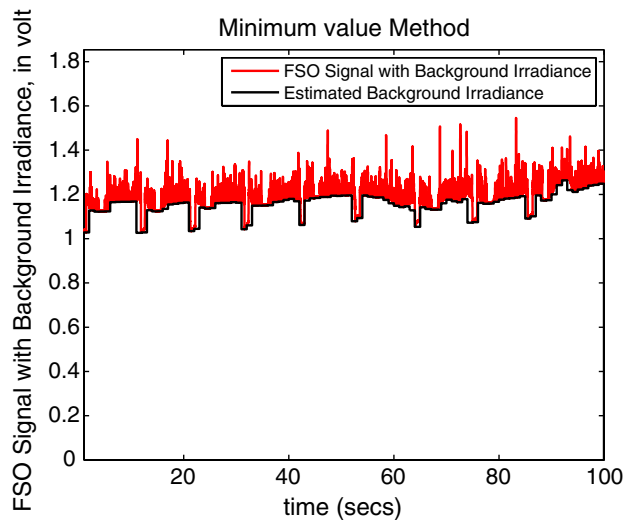

(a) Variable background estimation using MV method

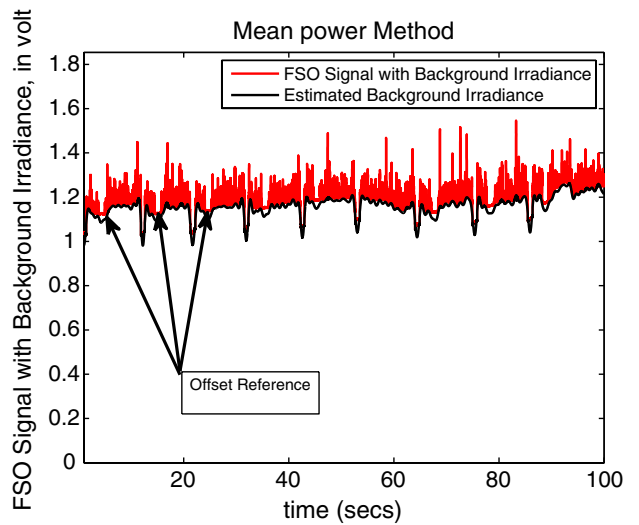

(b) Variable background estimation using MP method

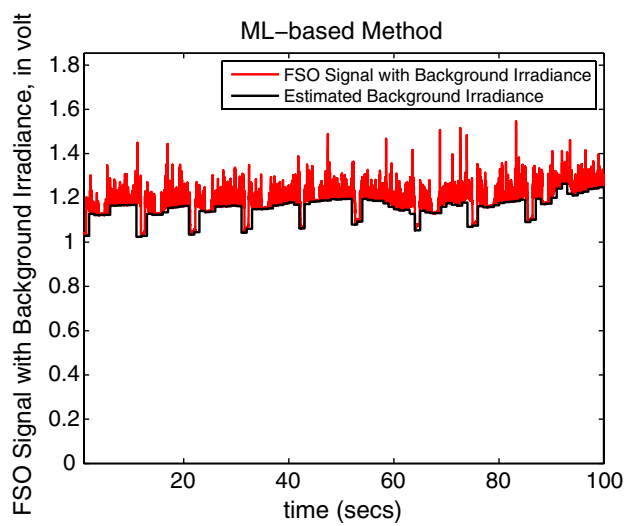

(c) Variable background estimation using MLbased method

Fig. 4. Estimation of background irradiance from frequently blocked experimental data. and Mawson Lakes in Adelaide, South Australia. Further details regarding the experiment can be found in [14]. Note that the sampling rate was $1 \mathrm{kHz}$.

Figure 3(a)-3(c) show the corresponding FSO channel, and estimated background irradiance by MV, MP, and ML-based methods, respectively. They also show the final vector after removing the background irradiance. We find that MP method gives better estimation results for a higher averaging period of 5-10 s. However, background irradiance can vary rapidly within $1-2 \mathrm{~s}$, which significantly affects its tracking ability. As the actual SI of the experimental data is unknown, it is not possible to comment on SI estimation accuracy.

In Fig. 3 the signal blockages can be observed as indicated by the arrows in Fig. 3(b). Note that we used two types of signal blockages: blocking the laser at the transmit side and blocking the receiver aperture. The latter is employed as a means of synchronizing the experiments, and the former is employed to provide a background reference to determine the estimation error. To determine the mean estimation error we repeated the experiment but with much smaller time intervals between the signal blockages. The results of this experiment are shown in Fig. 4 . Using these data we determined the mean estimation error by first estimating the background level using only the sample data corresponding to when the laser is blocked at the transmit end. We then estimate the background irradiance level immediately before and after the obstruction using the three methods described in Sections 3 and 4 . The observation interval for the MV and ML-based methods was set to $N=1000$ samples, $1 \mathrm{~s}$ worth of data. For the MP method a $1 \mathrm{~s}$ averaging period was used. The estimation error was then determined by subtraction of the background level during the obstruction from the estimates before and after the obstruction. This was then averaged over a number of blockages to determine the mean bias error, $\mu_{\epsilon}$, and RMS error $\sigma_{\epsilon}$. These results are summarized in Table 1 . It can be seen from Table 1 results that the ML estimator outperforms the $\overline{\mathrm{MV}}$ and MP estimators. The ML-based method has lower $\mu_{\epsilon}$ offset error before obstructions compared to the MV and MP methods. The standard deviation of the ML-based estimator is similar to the MV method before the obstructions. However, it is lower after the obstructions compared to other two methods. The MV method has lower $\mu_{\epsilon}$ offset error and $\sigma_{\epsilon}$ compared to the MP method before and after the obstructions.

\section{Conclusion}

We proposed three different estimators, namely MV, MP, and ML-based methods, to estimate the background irradiance and SI from FSO channel samples. Simulations were performed to compare the estimator performance in different SI regions. The MV and MP methods have low complexity compared to the ML-based method. The ML-based method gives 
better estimation performance from low- to high-SI regions compared to the other two methods. The MV method is useful in high-SI regions. The MP method can be used in low- to high-SI areas. It needs a longer averaging period and accurate estimation of MP from the obstructed period of optical data. Simulation and experimental trial results show that the proposed iterative ML-based method has low mean and standard deviation of offset error, and it can estimate the SI quite accurately compared to the other two methods. In addition, we demonstrated that the ML-based method is asymptotically unbiased and acheives an RMS error performance very close to the CRB, which is a lower bound on the RMS error performance of any estimator.

This work has been supported by the Sir Ross and Sir Keith Smith Fund, Cisco Systems, as well as the Defence Science and Technology Organisation (DSTO).

\section{Appendix A: Quasi-Newton BFGS Method}

Algorithm 2: Quasi-Newton BFGS Maximization [19,20]

1: Initialize $\hat{\boldsymbol{\theta}}_{0}, \boldsymbol{H}_{0}=\boldsymbol{I}$

2: while $k<N_{\text {iter }}$ do

3: Compute the direction vector, $\boldsymbol{d}_{k}=(\partial / \partial \boldsymbol{\theta}) \mathcal{L}\left(y ; P, z, \hat{\boldsymbol{\theta}}_{k}\right)$

4: Perform line search to optimize step size $0<\delta_{k}<1$.

5: Update the parameter estimates, $\hat{\boldsymbol{\theta}}_{k+1}=\hat{\boldsymbol{\theta}}_{k}-\delta_{k} \boldsymbol{H}_{k}^{-1} \boldsymbol{d}_{k}$.

6: Update the Hessian approximation,

$$
v_{k}=d_{k+1}-d_{k}
$$

$\boldsymbol{w}_{k}=\hat{\boldsymbol{\theta}}_{k+1}-\hat{\boldsymbol{\theta}}_{k}$

$\boldsymbol{H}_{k+1}=\boldsymbol{H}_{k}+\left(1+\left(\boldsymbol{v}_{k}^{T} \boldsymbol{H}_{k} \boldsymbol{v}_{k} / \boldsymbol{w}_{k}^{T} \boldsymbol{v}_{k}\right)\right)\left(\boldsymbol{w}_{k} \boldsymbol{w}_{k}^{T} / \boldsymbol{v}_{k}^{T} \boldsymbol{w}_{k}\right)$

$-\left(\boldsymbol{w}_{k} \boldsymbol{v}_{k}^{T} \boldsymbol{H}_{k} / \boldsymbol{w}_{k}^{T} \boldsymbol{v}_{k}\right)-\left(\boldsymbol{H}_{k} \boldsymbol{v}_{k} \boldsymbol{w}_{k}^{T} / \boldsymbol{w}_{k}^{T} \boldsymbol{v}_{k}\right)$.

$7: k=k+\mathbf{1}$

8: end while

\section{References}

1. L. C. Andrews and R. L. Phillips, Laser Beam Propagation through Random Media, 2nd ed. (SPIE, 2005).

2. A. K. Majumdar and J. C. Ricklin, Free-Space Laser Communications (Springer, 2008).

3. L. C. Andrews, R. L. Phillips, and C. Y. Hopen, Laser Beam Scintillation with Applications (SPIE Optical Engineering, 2001).

4. J. W. Strohbehn, Laser Beam Propagation in the Atmosphere (Springer-Verlag, 1978), vol. 25.
5. P. Beckmann, Probability in Communication Engineering (Harcourt, Brace \& World, 1967).

6. E. Jakeman and P. N. Pusey, "A model for non-Rayleigh sea echo," IEEE Trans. Antennas Propag. 24, 806-814 (1976)

7. M. A. Al-Habash, L. C. Andrews, and R. L. Phillips, "Mathematical model for the irradiance probability density function of a laser beam propagating through turbulent media," Opt. Eng. 40, 1554-1562 (2001).

8. A. Jurado-Navas, J. M. Garrido-Balsells, J. F. Paris, and A. Puerta-Notario, "A unifying statistical model for atmospheric optical scintillation," in Numerical Simulations of Physical and Engineering Processes (Intech, 2011), pp. 181-206.

9. E. Jakeman and P. N. Pusey, "Significance of $k$ distributions in scattering experiments," Phys. Rev. Lett. 40, 546-550 (1978).

10. F. S. Vetelino, C. Young, L. Andrews, and J. Recolons, "Aperture averaging effects on the probability density of irradiance fluctuations in moderate-to-strong turbulence," Appl. Opt. 46, 2099-2108 (2007).

11. N. Wang and J. Cheng, "Moment-based estimation for the shape parameters of the gamma-gamma atmospheric turbulence model," Opt. Express 18, 12824-12831 (2010).

12. J. Anguita, M. Neifeld, B. Hildner, and B. Vasic, "Rateless coding on experimental temporally correlated FSO channels," J. Lightwave Technol. 28, 990-1002 (2010).

13. S. D. Lyke, D. G. Voelz, and M. C. Roggemann, "Probability density of aperture-averaged irradiance fluctuations for long range free space optical communication links," Appl. Opt. 48, 6511-6527 (2009).

14. D. Giggenbach, W. Cowley, K. Grant, and N. Perlot, "Experimental verification of the limits of optical channel intensity reciprocity," Appl. Opt. 51, 3145-3152 (2012).

15. A. Papoulis, Probability, Random Variables, and Stochastic Processes (McGraw-Hill, 1991).

16. L. C. Andrews, R. L. Phillips, C. Y. Hopen, and M. A. Al-Habash, "Theory of optical scintillation," J. Opt. Soc. Am. A 16, 1417-1429 (1999).

17. H. A. David, Order Statistics (Wiley, 2003).

18. S. M. Kay, Fundamentals of Statistical Signal Processing: Estimation Theory (Prentice-Hall, 1993).

19. S. S. Rao, Engineering Optimization Theory and Practice (Wiley, 1996).

20. A. Dogandzic and J. Jin, "Maximum likelihood estimation of statistical properties of composite gamma-lognormal fading channels," IEEE Trans. Signal Process. 52, 2940-2945 (2004).

21. M. Abramowitz and I. A. Stegun, Handbook of Mathematical Functions with Formulas, Graphs and Mathematical Tables (Dover, 1972)

22. A. Khatoon, W. Cowley, and N. Letzepis, "Channel measurement and estimation for free space optical communications," in Australian Communications Theory Workshop (AusCTW), 2011 (IEEE, 2011), pp. 112-117. 